\title{
ELECTRONIC HEALTH RECORD USING BLOCKCHAIN AND OFF CHAIN STORAGE: A SYSTEMATIC REVIEW
}

\author{
Pranalini Joshi ${ }^{1}$, Dr.Prasad Gokhale ${ }^{2}$ \\ ${ }^{1}$ Research Scholar, Vishwakarma University, Pune, India \\ ${ }^{2}$ Professor, Vishwakarma University, Pune, India \\ pranalini.ketkar@gmail.com,prasad.gokhale@vupune.ac.in
}

\begin{abstract}
The Blockchain was introduced by Bitcoin [1] for maintaining financial transaction; many researches are going on, to extend the use of blockchain technology in non-financial application. Healthcare domain is a promising industry in which blockchain can have important influence. This paper focus on a systematic review about existing and ongoing research in the field of storing electronic health record (EHR) using blockchain technology. This paper also presents use of blockchain framework in healthcare domain, its challenges and further research.
\end{abstract}

Index Terms - electronics health record, Blockchain, on chain storage, off chain storage

\section{INTRODUCTION}

Healthcare is a data-sensitive domain where a large amount of data is produced, disseminated, deposited, and gets retrieved daily. For example, whenever a patient visits hospital, some data will get created which may be used by multiple doctors i.e. physicians/ surgeons etc. within same hospital or hospitals within networks. [3]

Healthcare data contain individual and sensitive information that may attract to cyber criminals. Hence ensuring the security of the electronic health record (EHR) is crucial and challenging due to the exchange of information between the multiple stockholders like doctors, insurance company etc. Furthermore, the confidentiality and reliability of healthcare data must be protected from external attackers and also from illegal access attempts from inside the network or ecosystem. [3]

Therefore, to protect the data from the misuse and also to maintain a definite trust between the different stakeholders like doctor, Patient, Insurance companies etc. A system is needed where the transactions, complete access and storage management was taken over by the technology and blockchain technology can be used for the same.

\section{BLOCKCHAIN TECHNOLOGY}

Blockchain is decentralized and distributed ledger technology. The blockchain is nothing but chain of blocks, where every block represents a set of transactions. Every Record is referred as ledger and data exchange refers as transaction. A Blockchain makes the use of distributed system to validate each transaction. Very important property of blockchain is that it is a immutable technology i.e whenever any transaction or blocks get added in blockchain it remain unaltered and cant not edit. In addition to this following are few features of blockchain technology

Decentralized: Every node has one copy of data; hence failure of one node does not affect the organization.

Secure: All the data is hashed with cryptography i.e. using key and hence it is secured

Irreversible: Once transaction is stored in Blockchain, it cannot be rollback

Reliable: As Blockchain is decentralized, even if a single node gets fail, it does not affect the entire system, thus makes Blockchain network reliable

Efficiency: As transactions are not controlled by any central authority, it takes less processing time

Like any other technology, Blockchain is evolved from Blockchain 1.0 to 3.0. Bitcoin implemented cryptocurrency which is used for financial transaction is always referred as Blockchain 1.0. Blockchain 2.0 introduced concept of Smart contracts and smart properties. Smart Properties means digital assets, ownership of these smart properties can be controlled by a blockchain-based platform. The smart contracts are the software programs which decide the rules to controlled and managed smart properties. Ethereum, QTUM , Ethereum Classic are some examples of Blockchain 2.0. Solidity is popular language to write smart contracts. Blockchain 3.0 is the third generation of blockchain technology All the non-financial applications blockchain are implemented using Blockchain 3.0 Application such as supply chain management, Electronics health records, Document verification fall under this category [4]. Step by step working of blockchain technology is shown in figure 1. 


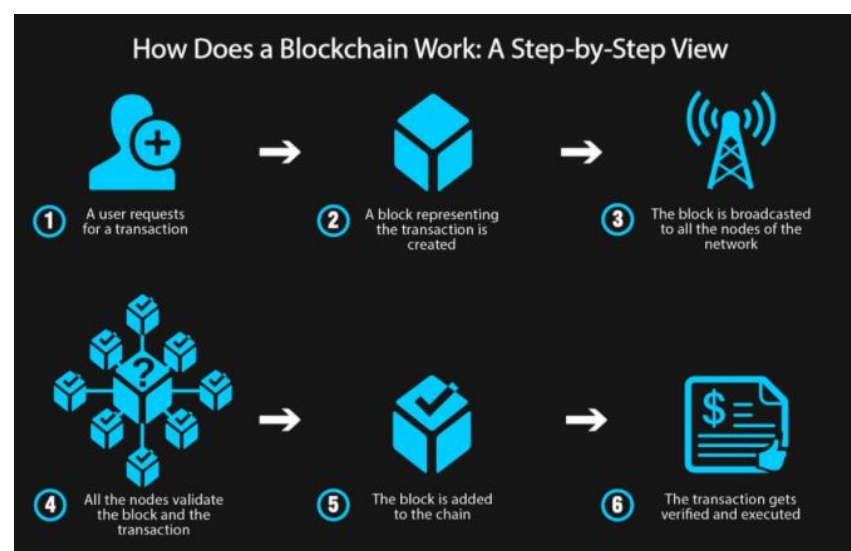

Figure 1: Working of Blockchain Technology [58]

\section{BLOCKCHAIN IN HEALTHCARE}

To get a clear idea and to identify the scope about storing personalized or electronic health record using blockchain technology, the systematic literature review has been performed. Almost 60 Papers from IEEE, Science Direct, PUBMED, various medical Journals databases are reviewed. Few papers were related to introduction of blockchain technology, IPFS, Etherium and Heperledger. Some papers were survey papers and in some papers a system to store electronic health record was either proposed or implemented. Only those papers which has either proposed or implemented a system are used in this review for analysis. Basic terminology on basis of which analysis of these literature have done are

1. Blockchain Platform and Consensus mechanism Used

2. Scalability or storage Schemes

3. Data Provenance

4. Access Control Methods

This paper also focuses on Opportunities and challenges of using blockchain in healthcare domain.

\subsection{Blockchain Platform and Consensus mechanism} Used

Etherium[11] and hyperledger[55] are the two major types of blockchain platforms which are used to build non-financial applications using blockchain .Very few papers showed use of bitcoin platform . Fig.1 shows File structure and architecture of Etherium and Hyperledger blockchain. Etherium, Bitcoin are public blockchain platform whereas Hyperledger is framework to build private or consortium blockchain. Blockchain architecture of Etherium and hyperledger is shown in Figure 2.

MedRec is model proposed in [42] uses Etherium client and it requires miners in blockchain, which may lead to a new problem i.e. shortage of Human Resource Quality [22]. In this model whenever patient wishes to share information they have to spend ether. Therefore Selection of blockchain platform is very important while using blockchain technology in non-financial applications
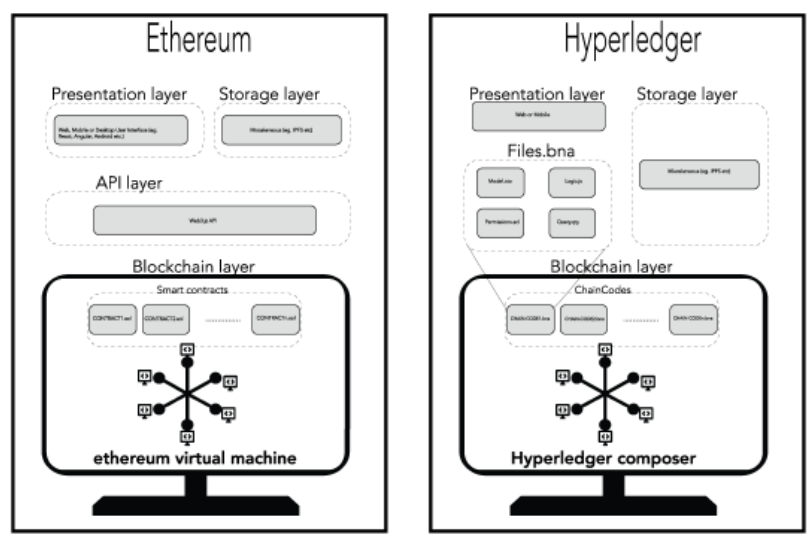

Figure 2: Etherium and Heperledger blockchain architecture and file structure [8]

Blockchain can also be classified on basis of consensus mechanism used by network. Consensus algorithm is the mechanism to check valid transactions and blocks. Only valid transactions or blocks are added in blockchain. Process of consensus is also called as mining and nodes who take parts in mining process are called as miners. Consensus algorithms are primarily classified into two groups, first is Proof Based algorithms and second is Vote Based algorithms [8]. Proof of work (PoW), Proof of stack $(\mathrm{PoS})$ are popular proof based algorithms and Practical Byzantine Fault Tolerance (PBFT) is very popular vote based consensus algorithm. Table 1 [57] shows the comparatives study of PoW, PoS and PBFT.

System Proposed by Hao Guo [36] uses concept of Hyperledger Composer Fabric [20] which is a blockchain implementation platform and supports plug and play consensus mechanism.

\begin{tabular}{|l|l|l|l|}
\hline \multicolumn{1}{|c|}{ Property } & \multicolumn{1}{c|}{ PoW } & \multicolumn{1}{c|}{ PoS } & \multicolumn{1}{c|}{ PBFT } \\
\hline $\begin{array}{l}\text { Node } \\
\text { Management }\end{array}$ & Open & Open & Permissioned \\
\hline $\begin{array}{l}\text { Energy } \\
\text { Consumption }\end{array}$ & High & Medium & Low \\
\hline $\begin{array}{l}\text { Tolerated } \\
\text { Power of } \\
\text { Adversary }\end{array}$ & $<25 \%$ & $\begin{array}{l}<51 \% \\
\text { Stake }\end{array}$ & $\begin{array}{l}<33.3 \text { faulty } \\
\text { Replicas }\end{array}$ \\
\hline Example & Bitcoin [1] & Peercoin & $\begin{array}{l}\text { Hyperledger } \\
\text { Fabric [55] }\end{array}$ \\
\hline
\end{tabular}

Table 1: Consensus Mechanism Comparison [57]

\subsection{Scalability and Storage Scheme}


Property of a system which handles growing amount of work by addition of resources to the system is known as scalability. One of the major challenge in Blockchain is Scalability. As blockchain is platform created for financial transaction (cryptocurrency) by bitcoin, size of transaction is very small. It is very ineffective to store large files on blockchain as it slow down block processing speeds and leads to the problem of scalability. Abstract data types like MRI, X-Ray images etc cannot directly store in blockchain. This type of data would require links to a separate location. By considering technical and confidentiality constraints data should be stored in ON-chain and OFF-chain formats. [54].Many papers have tried to solve this issue of scalability have solved using different Off-Chain storage.

In most of proposed system a centralized storage like cloud, SQLite and central server is used to store patients data as off-chain storage which again has a chance of single point failure .To avoid this a concept of IPFS is used in many papers like [13][15]. IPFS is inter planetary file system. It is a peer-to-peer (P2P) distributed storage system in which huge volumes of records can get easily stored. For every file, IPFS generates a unique hash and store that hash in a table called as Distributed Hash Table (DHT) and removes duplicate files using version-control history [23].

IPFS generates a unique hash for every file and uses content based addressing instead of location based addressing for file retrieval .It has many features such as high throughput, Security due to mapping of transaction with hash and concurrent access to transaction by peers in network.[15]

\subsection{Data Provenance}

Data Provenance in a system provides a detailed picture of how the data was collected, where this data gets stored and how it is being used. In other words, data provenance is nothing but "showing your work" in a database. This historical record of information can then be reliable source for data validation and audit purposes. The historical records of data and their origins are always referred as Data Provenance [26].

Most of the paper reviewed, proposed systems have properly shown data provenance. In various proposed system's data is either collected using wearable device or sensors inserted in human body or manually from information provided by patients.

Table 2 shows Off-chain Storage, consensus mechanism, Data Provenance used by various proposed system.

\subsection{Access control}

Access Control means systems perform identification, authentication and authorization of users and entities by evaluating required login credentials. Using blockchain technology two types of access control can occur

\section{Role based access control}

\section{Attribute Based}

In terms of Electronic health record, the term access control refers to which user will have access to individual / Patient data. Most of the proposed system is patient centric where patients have full access to his own data. Smart contracts have played very important role to specify access in various system.[13] [36] are role based access control where third party would not be able to access a particular data as it is unauthorized user for that particular data .

\begin{tabular}{|l|l|l|l|}
\hline $\begin{array}{l}\text { Ref. } \\
\text { NO }\end{array}$ & $\begin{array}{l}\text { Off-chain } \\
\text { Storage used }\end{array}$ & $\begin{array}{l}\text { Consensus } \\
\text { Mechanism } \\
\text { used }\end{array}$ & $\begin{array}{l}\text { Data } \\
\text { Provenance }\end{array}$ \\
\hline$[5]$ & Cloud & POS & YES \\
\hline$[42]$ & SQLite & POW & YES \\
\hline$[7]$ & Central Server & PBFT & YES \\
\hline$[12]$ & $\begin{array}{l}\text { Fast } \\
\text { Healthcare } \\
\text { Interoperability } \\
\text { Resources (FHIR) }\end{array}$ & $\begin{array}{l}\text { Multichain } \\
\text { Algorithms }\end{array}$ & YES \\
\hline$[13]$ & $\begin{array}{l}\text { Inter Planetary File } \\
\text { System [IPFS ] }\end{array}$ & POW & YES \\
\hline$[15]$ & $\begin{array}{l}\text { Inter Planetary File } \\
\text { System [IPFS ] }\end{array}$ & POW & YES \\
\hline$[36]$ & Edge Node & --- & YES \\
\hline
\end{tabular}

Table 2: Analysis of various proposed system

\section{CHALLENGES OF BLOCKCHAIN ADAPTATION IN HEALTHCARE:}

Though blockchain in healthcare has attracted many attention, there are various challenges that needs to be improved while accepting blockchain adoption

\section{Public Vs Private Vs Consortium Blockchain -}

Blockchain technology can be categories into 3 categories

Public Blockchain: In public Blockchain, all records can be viewed by the public and everyone could read, write and participate in Blockchain consensus process. It is also known as permission less Blockchain In public Blockchain, no one has control over the network, also once data get validated it cannot changed.

Private Blockchain: It is also called as permissioned block chain. It is specific for an organization 
and only nodes from those organization are allowed to read, write and participate in consensus process. It is like centralized network which is completely managed by one organization.

Consortium Blockchain: It is specific for group of organizations and nodes from those organizations are allowed to read, write and participate in consensus process.

Though Public Blockchain like bitcoin implementation provides best data integrity due to multiple copies and redundancy of the ledger.

Main Challenge in public Blockchain is Security that means data must be protected and should not open in public domain. Private Blockchain overcome this challenge and hence are popular than public blockchain.

However implementations of private blockchain navigate the blockchain development away from a decentralized to centralized system and the original moto of Blockchain was to avoid centralized systems.[6].

Most of proposed systems have suggested using Private Blockchain/ consortium blockchain like Etherium or Hyperledger to get more security and confidentiality. [25][36] have used hyperledger Fabric where [13] is using Etherium in their proposed prototype. Table 3 [57] shows comparison of private, public and consortium blockchain

\begin{tabular}{|c|c|c|c|}
\hline Property & $\begin{array}{l}\text { Public } \\
\text { Blockchain }\end{array}$ & $\begin{array}{l}\text { Consortium } \\
\text { Blockchain }\end{array}$ & $\begin{array}{l}\text { Private } \\
\text { Blockchain }\end{array}$ \\
\hline $\begin{array}{l}\text { Consensus } \\
\text { Determinatio } \\
\text { n }\end{array}$ & All Miners & $\begin{array}{l}\text { Selected set } \\
\text { of nodes }\end{array}$ & $\begin{array}{l}\text { One } \\
\text { organization }\end{array}$ \\
\hline $\begin{array}{l}\text { Read } \\
\text { Permission }\end{array}$ & public & $\begin{array}{l}\text { Public or } \\
\text { restricted }\end{array}$ & $\begin{array}{l}\text { Public or } \\
\text { restricted }\end{array}$ \\
\hline Immutability & $\begin{array}{l}\text { Nearly } \\
\text { impossible }\end{array}$ & $\begin{array}{l}\text { Could be } \\
\text { tampared }\end{array}$ & $\begin{array}{l}\text { Could be } \\
\text { tampared }\end{array}$ \\
\hline Efficiency & Low & High & High \\
\hline Centrality & No & Partial & Yes \\
\hline $\begin{array}{l}\text { Consensus } \\
\text { Process }\end{array}$ & $\begin{array}{l}\text { Permission } \\
\text { less }\end{array}$ & Permissioned & $\begin{array}{l}\text { Permissione } \\
\text { d }\end{array}$ \\
\hline
\end{tabular}

Table 3: Different types of Blockchain Overview

2. Interoperability: Interoperability is another challenge in field of Blockchain. Interoperability of healthcare records is the degree to which the clinical data can be conveyed across institutional boundaries. Due to the complexities of data in the healthcare domain, this is inherently difficult to achieve interoperability [12]

Interoperability is ability of various related systems, devices or applications to connect in a coordinated manner within and across organizational boundaries to exchange, access and cooperatively use data among stakeholders with aim of optimizing health of individuals and populations [26]

Interoperability can be improved using blockchain technology by exchanging data across multiple systems and can be accessed simultaneously. Improved Interoperability may lead to improved health outcome [37]

In Healthcare domain, data can be shared within institutional boundaries or across institutional boundaries. Most of the proposed systems share the data within institutional boundaries or patient's/ User centric where patients have full right to give access of his own data.

3. Legal Issue: Third important challenge is legal issues . The legal issues are related to the law that been implement in IT in order to protect the personal data but by the aggressive protection that cause the difficult to the users to access the PHRs.[2]

Many proposed system have not talk on this topic. But through proper agreement this problem can be solved

\section{APPLICATIONS OF BLOCKCHAIN IN HEATH ECOSYSTEM}

According to [14], Blockchain technology can not only be used to store Electronic health record but also can be used in various domains like Detection of Medical Fraud, Neuroscience and Clinical Research, Pharmaceutical Industries etc. Various Applications wher blockchain can be used are shown in figure 3.

Where and Why Blockchain can be used in Healthcare?

Clinical Research: as blockchain can be used as decentralized framework, group of researcher can used it for secured data sharing.

Fraud Detection: As transactions in blockchain are immutable, No one can update or duplicate transaction and therefore frauds can get identified easily

Pharmaceutical Industries: For supply chains where participants are not known or trusted, blockchain technology can add trust, transparency, and traceability. 


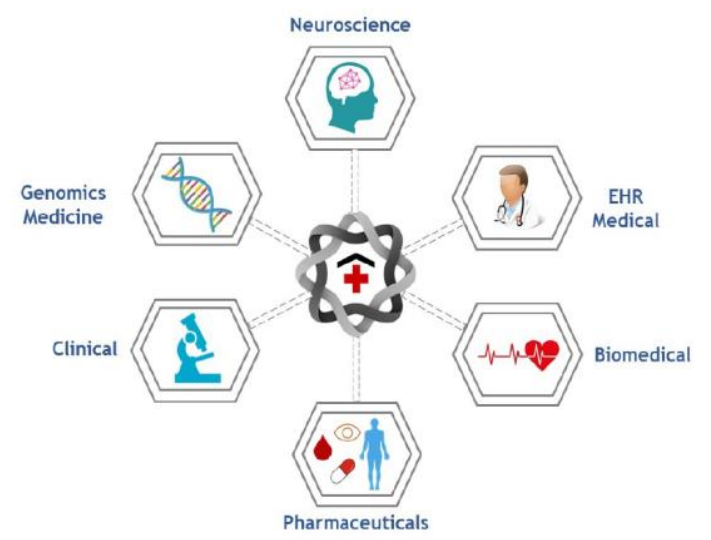

Figure 3: Application of blockchain in Healthcare [14]

\section{CONCLUSION}

Blockchain signifies a new form of technology which can be used in healthcare domain. There is scope to explore utilization of blockchain in healthcare domain such as the incorporation of hospitals, healthcare start-up, Personal health insurance etc. Government can also use it to provide various heath schemes.

During literature review it is observed that the current literature is lacking in the above stated application context and statistical comparisons with traditional systems or other proposed systems have not been made anywhere. In future, research agenda should be extended to concentrate on the mentioned challenges.

\section{REFERENCES}

[1] Nakamoto, S. (2008). Bitcoin: A peer-to-peer electronic cash system.

[2] N.M. Yaacob, A.S. Shibghatullah, M.K. Ghani and L. Salahuddin ," A Review on Electronic Personalized Health Records “,Journal of Telecommunication, Electronic and Computer Engineering, Volume 10 , issue 10.

[3] Blockchain: A Panacea for Healthcare Cloud- Based, Data Security and Privacy ?, IEEE Cloud Computing Copublished by the IEEE CS and IEEE Com Soc January/February 2018

[4] Cornelius C. Agbo *, Qusay H. Mahmoud and J. Mikael Eklund,Review Blockchain Technology in Healthcare: A Systematic Review, www.mdpi.com/journal/healthcare, 4 April 2019

[5] Sabyasachi Chakraborty, Satyabrata Aich, Hee-Cheol Kim A Secure Healthcare System Design Framework Using Blockchain Technology, International Conference On Advanced Communications Technology(Icact), February 2019

[6] Towards Secure and Smart Healthcare in Smart Cities Using Blockchain

[7] Koosha Mohammad Hossein, Mohammad Esmaeil Esmaeili, Tooska Dargahi Blockchain-Based PrivacyPreserving Healthcare Architecture “, 2019 IEEE Canadian
Conference of Electrical and Computer Engineering (CCECE )

[8] Emeka Chukwu And Lalit Garg , “ A Systematic Review of Blockchain in Healthcare: Frameworks, Prototypes, and Implementations "IEEE Access Year: 2020|Volume: 8|Journal Article |Publisher: IEEE [9] Zonyin Shae, Jeffrey J.P.Tsai "On the Design of a Blockchain Platform for Clinical Trial and Precision Medicine "2017 IEEE 37th International Conference on Distributed Computing Systems

[10] Dinh C. Nguyen , Pubudu N. Pathirana, Ming Ding, And Aruna Seneviratne Blockchain For Secure Ehrs Sharing Of Mobile Cloud Based E-Health Systems, Ieee Access Journal May 17, 2019 (Special Section On Healthcare Information Technology For The Extreme And Remote Environments )

[11] By Vitalik Buterin “ A Next Generation Smart

Contract \&

decentralized Application Platform" (2013) Whitepaper

[12] Kevin Peterson, Rammohan Deeduvanu, Pradip Kanjamala, and Kelly Boles, A Blockchain-Based Approach to Health Information Exchange Networks, Mayo Clinic

[13] Ayesha Shahnaz 1, Usman Qamar1, And Ayesha Khalid, Using Blockchain For Electronic Health Records, Ieee Access Journal October 9, 2019, Digital Object Identifier 10.1109/Access.2019.2946373

[14] Asad Ali Siyal , Aisha Zahid Junejo, Muhammad Zawish , Kainat Ahmed, Aiman Khalil and Georgia Soursou ," Applications of Blockchain Technology in Medicine and Healthcare: Challenges and Future Perspectives" Article

[15] Randhir Kumar, Rakesh Tripathi , Ningrinla Marchang "Distributed O.-chain Storage of Patient Diagnostic Reports in Healthcare System using IPFS and Blockchain “ , 2020 12th International Conference on Communication Systems \& Networks (COMSNETS)

[16] Randhir Kumar, Rakesh Tripathi “ Implementation of Distributed File Storage and Access Framework using IPFS and Blockchain "2019 Fifth International Conference on Image Information Processing (ICIIP)

[17] Mathis Steichen, Beltran Fiz, Robert Norvill, Wazen Shbair and Radu State, "Blockchain-Based, Decentralized Access Control for IPFS “ 2018 IEEE Confs on Internet of Things, Green Computing and Communications, Cyber, Physical and Social Computing, Smart Data, Blockchain, Computer and Information Technology, Congress on Cybermatics

[18] Qiuhong Zhenge, Yi Li, Ping Chen An innovative IPFS -Based Storage model for blockchain 2018 IEEE/WIC/ACM International Conference on Web Intelligence (WI) 
[19] Jiajie Shen, Yi Li, Yangfan Zhou, and Xin Wang "Understanding I/O Performance of IPFS Storage: A Client's Perspective "2019 IEEE/ACM 27th International Symposium on Quality of Service (IWQoS)

[20] Hyperledger Fabric. [Online]. Available: https:// www. hyperledger. org/projects/fabric

[21] Wei Liu, Qinyong Yu, Zesong L , Zeyuan , Yu Su, Jian Zhou "A Blockchain-Based System for Anti-Fraud of Healthcare Insurance" 2019 IEEE 5 th International Conference on Computer and Communications

[22] Mogi Jordan Christ, Rahmanto Nikolaus Permana Tri, Wiranto Chandra Wang Gunawan, "Exploring Blockchain in Healthcare Industry"

[23] IPFS - Content Addressed, Versioned, P2P File System (IPFS white paper) arXiv preprint arXiv:1407.3561

[24] Rui Guo, Huixian Shi , Dong Zheng, Chunming Jing, Chaoyuan Zhuang, And Zhengyang Wang1, "Flexible And Efficient Blockchain-Based Abe Scheme With MultiAuthority For Medical On Demand In Telemedicine System" IEEE Access Year: 2019|Volume: 7|Journal Article | Publisher: IEEE.

[25] Tomas Mikula and Rune Hylsberg Jacobsen, "Identity and Access Management with Blockchain in Electronic Healthcare Records" 2018 21st Euromicro Conference on Digital System Design (DSD)

[26]Anton hasselgren , Katina Kralevska,Danilo Gligoroski, Sindre A. Pedersen , Arild Faxvaag ," Review Article on Blockchain in healthcare and health sciences - A scoping review", International Journal of Medical Informatics , 134 (2020)

[27] Thein Than Thwin and Sangsuree Vasupongayya , “ Research Article blockchain-Based Access Control Model to Preserve Privacy for Personal Health Record Systems “ Willey, Published 25 June 2019

[28] Sara Rouhani and Ralph Deters. 2018. Blockchain based access control systems: State of the art and challenges. International Conference on Web Intelligence (Web Intelligence '19 October 14-17). ACM,New York, NY, USA, 6 pages, https://doi.org/10.1145/1122445.1122456

[29] Kaihua Qin, Arthur Gervais ,“ An overview of blockchain scalability, interoperability and sustainability"

[30] G. Foroglou and A.-L. Tsilidou, "Further applications of the blockchain, " 2015.

[31] K. Christidis and M. Devetsikiotis, "Blockchains and Smart Contracts for the Internet of Things," IEEE Access, vol. 4, pp. 2292-2303, 2016

[32] Sandi Rahmadika and Kyung-Hyune "Blockchain technology for providing an architecture model of decentralized personal health information "International Journal of Engineering Business Management Volume 10: $1-12$
[33] Haidich AB, Review article on "Meta-analysis in medical research “,HIPPOKRATIA 2010, 14 (Suppl 1): 2937

[34] D. D. F. Maesa, P. Mori, and L. Ricci, "Blockchain based access control," in IFIP International Conference on Distributed Applications

and Interoperable Systems. Springer, 2017, pp. 206-220.

[35] Hannah S Chen†, Juliet T Jarrell†, Kristy A Carpenter, David S Cohen, Xudong Huang, "Blockchain in Healthcare: A Patient-Centered Model “ Biomed J Sci Tech Res. 2019 ; 20(3): 15017-15022.

[36] Hao Guo,Wanxin Li, Mark Nejad, Chien-Chung Shen1 , "Access Control for Electronic Health Records with Hybrid Blockchain-Edge Architecture “, 2019 IEEE International Conference on Blockchain

[37] Implementing Blockchains for Efficient Health Care: Systematic Review , JOURNAL OF MEDICAL INTERNET RESEARCH, Anuraag A Vazirani1; Odhran O'Donoghue1; David Brindley1

[38] Mohamed Yaseen Jabarulla, and Heung-No Lee , "Blockchain-Based Distributed Patient-Centric Image Management system."

arXiv:2003.08054

[39] Muhammad Umar Javed 1, Mubariz Rehman 1, Nadeem Javaid 1,*, Abdulaziz Aldegheishem 2, Nabil Alrajeh 3 and Muhammad Tahir, Article "BlockchainBased Secure Data Storage for Distributed Vehicular Networks" Appl. Sci. 2020, 10, 2011; doi:10.3390/app10062011

[40] Veneta Aleksieva, Hristo Valchanov and Anton Huliyan ," Implementation of Smart-Contract, Based on Hyperledger Fabric Blockchain “,2020-21st International Symposium on Electrical Apparatus \& Technologies (SIELA)

[41] Ron Ribitzky, James St. Clair, David I. Houlding, Chrissa T. McFarlane, Brian Ahier, Michael Gould, Heather L. Flannery,Erik Pupo,Kevin A. Clauson ," Pragmatic, Interdisciplinary Perspectives on Blockchain and Distributed Ledger Technology: Paving the Future for Healthcare"

[42] Asaph Azaria, Ariel Ekblaw, Thiago Vieira and Andrew Lippman "MedRec: Using Blockchain for Medical Data Access and Permission Management "2016 2nd International Conference on Open and Big Data

[43] Tsung-Ting Kuo and Lucila Ohno-Machado, "ModelChain: Decentralized Privacy-Preserving Healthcare Predictive Modeling Framework on Private Blockchain Networks

arXiv: 1802.01746

[44] Xueping Liang,Juan Zhao, Sachin Shetty4, Jihong Liu, Danyi Liy ," Integrating Blockchain for Data Sharing and Collaboration in Mobile Healthcare Applications " 2017 IEEE 28th Annual International Symposium on Personal, Indoor, and Mobile Radio Communications (PIMRC) 
[45] Document on "Reimagining health information exchange in INDIA using Blockchain “ April 2019, by PricewaterhouseCoopers Private Limited

[46] Paul C. Tang, Joan S. Ash, David W. Bates, J. Marc Overhage, Daniel Z. Sands Personal Health Records: Definitions, Benefits, And Strategies For Overcoming Barriers To Adoption, White Paper, Journal Of The American Medical Informatics Association Volume 13 Number 2 Mar / Apr 2006

[47] Sofia Alexaki, George Alexandris, Vasilis Katos, Nikolaos E. Petroulakis , " Blockchain-based Electronic Patient Records for Regulated Circular Healthcare Jurisdictions" 2018 IEEE 23rd International Workshop on Computer Aided Modeling and Design of Communication Links and Networks (CAMAD)

[48] Rui Guo ,Huixian Shi , Dong Zheng, Chunming Jing , Chaoyuan Zhuang, And Zhengyang Wang "Flexible and Efficient Blockchain-Based ABE Scheme With MultiAuthority for Medical on Demand in Telemedicine System “ IEEE Access, June 28, 2019.

[49] Yongle Chen, Hui Li* , Kejiao Li and Jiyang Zhang, “ An improved P2P File System Scheme based on IPFS and Blockchain “, 2017 IEEE International Conference on Big Data (BIGDATA) .

[50] Blockchain-Based Framework For Data Storage In Peer-To-Peer Scheme Using Interplanetary File System Randhir Kumar And Rakesh Tripathihandbook Of Research On Blockchain Technology, Chapter 2, Page No-35-59

[51] Miltiadis D. Lytras, Akila Sarirete2 and Vassilios Stasinopoulos " Policy implications for smart healthcare: the international collaboration dimension, Innovation in Health Informatics, 2020 Elsevier, Chapter 17, Page No-395-402

[52] Adya Kiran, Samvid Dharanikota, Annappa Basava “ Blockchain based Data Access Control using Smart Contracts "TENCON 2019 - 2019 IEEE Region 10 Conference (TENCON)

[53] Claude Pirtle, Jesse Ehrenfeld "Blockchain for Healthcare: The Next Generation of Medical Records?" Journal of Medical Systems (2018) 42: 172, Springer.com

[54] RJ Krawiec, Dan Housman, Mark White, Mariya Filipova, Florian Quarre, Dan Barr, Allen Nesbitt, Kate Fedosova, Jason Killmeyer, Adam Israel, Lindsay Tsai ," Blockchain: Opportunities for Health Care "White paper, Deolite

[55] Elli Androulaki ,Christian Cachin ,Christopher Ferris , Gennady Laventman ,Srinivasan Muralidharan ,Chet Murthy, Binh Nguyen ,Manish Sethi ,Gari Singh ,Keith Smith ,Alessandro Sorniotti ,Chrysoula Stathakopoulou , " Hyperledger Fabric: A Distributed Operating System for Permissioned Blockchains “, EuroSys '18: Proceedings of the Thirteenth EuroSys Conference April 2018 Article No.: 30 Pages $1-15$
[56] Mathis Steichen, Beltran Fiz, Robert Norvill, Wazen Shbair and Radu State ,"Blockchain-Based, Decentralized Access Control for IPFS" 2018 IEEE International Conference on Internet of Things (iThings) and IEEE Green Computing and Communications (GreenCom) and IEEE Cyber, Physical and Social Computing (CPSCom) and IEEE Smart Data (SmartData)

[57] Zibin Zheng, Shaoan Xie, Hongning Dai, Xiangping Chen, Huaimin Wang. "An Overview of Blockchain Technology: Architecture, Consensus, and Future Trends", 2017 IEEE International Congress on Big Data (BigData Congress), 2017

[58] https://101blockchains.com 